\title{
Modelling latent carbon emission prices: theory and practice
}

\author{
Chia-Lin Chang ${ }^{a}$ and Michael McAleer ${ }^{b, c, d, e, f}$ \\ ${ }^{a}$ Department of Applied Economics, Department of Finance, National Chung Hsing University, Taiwan \\ ${ }^{b}$ Department of Finance, Asia University, Taiwan \\ ${ }^{c}$ Discipline of Business Analytics, University of Sydney Business School, Australia \\ ${ }^{d}$ Econometric Institute, Erasmus School of Economics, Erasmus University Rotterdam, the Netherlands \\ ${ }^{e}$ Department of Economic Analysis and ICAE, Complutense University of Madrid, Spain \\ ${ }^{f}$ Institute of Advanced Sciences, Yokohama National University, Japan \\ Email: michael.mcaleer@gmail.com
}

\begin{abstract}
Climate change and global warming are significantly affected by carbon emissions that arise from the burning of fossil fuels, specifically coal, oil and gas. Accurate prices are essential for purposes of measuring, capturing, storing and trading in carbon emissions at the regional, national, and international levels, especially as carbon emissions can be taxed appropriately when the price is known and widely accepted. The paper uses a novel KLEM production function approach to calculate the latent carbon emission prices, where carbon emission is the output and capital (K), labour (L), energy (E) (or electricity), and materials (M), are the inputs into the production process. The variables $\mathrm{K}, \mathrm{L}$ and $\mathrm{M}$ are essentially fixed on a daily or monthly basis, whereas E can be changed more frequently, such as daily or monthly, so that changes in carbon emissions depend on changes in E. If prices are assumed to depend on average cost pricing, the prices of carbon emissions and energy may be approximated by an energy production model with a constant factor of proportionality, so that carbon emission prices will be a function of energy prices. Using this novel modelling approach, the paper estimates carbon emission prices for Japan using seasonally adjusted and unadjusted monthly data on the volumes of carbon emissions and energy, as well as energy prices, from December 2008 to April 2018. The econometric models show that, as sources of electricity, the logarithms of coal and oil, though not LNG, are statistically significant in explaining the logarithm of carbon emissions, with oil being more significant than coal.
\end{abstract}

Keywords: Latent carbon emission prices, fossil fuels, energy, KLEM production function, average cost pricing 


\section{INTRODUCTION}

Climate change and global warming are two of the most important environmental issues presently facing the international community. Global warming is typically defined as the observed century-scale rise in the average temperature of the earth's climate system and its related effects, while climate change refers to the change in the statistical distribution of weather patterns, specifically average weather conditions, over an extended period, such as a century. Unlike misleading and confused comments made recently by a leader of the so-called free world, neither climate change nor global warming refers to changing weather conditions on a daily or otherwise high frequency data observation basis (see Allen and McAleer (2018)).

Although the clear leader in the battle to reduce and mitigate the effects of carbon emissions is the European Union, China has also started to mitigate the effects of greenhouse gases through the development of domestic regional carbon emission trading markets, with the goal of extending it to the national level in the near future. The creation of such markets that trade in carbon emissions is intended to establish prices that can be used to buy and sell carbon emissions, and to reduce the harmful effects of such emissions on the social and physical environment.

Accurate prices are essential for purposes of measuring, capturing, storing and trading in carbon emissions at the regional, national, and international levels, especially as carbon emissions can be taxed appropriately when the price is known and widely accepted. In the absence of an active trading market for carbon emissions, the prices are typically decided through arbitrary administrative deliberations, and hence are not efficient or even necessarily accurate. In this sense, carbon emission prices are latent.

The purpose of the paper is to price the latent carbon prices through a novel econometric approach that can be used to calculate realized latent prices of carbon emissions as a function of capital (K), labour (L), energy (E) (that is, electricity), and materials (M) inputs. The inputs K, L and M are typically fixed on a daily, monthly or otherwise high frequency data observation interval, whereas E can be changed with high data frequency, such as daily or monthly, so that changes in carbon emissions will depend on changes in E. If prices are assumed to depend on average cost pricing, the prices of carbon emissions and energy may be approximated by an energy production model with a constant factor of proportionality, so that carbon emission prices will be a function of energy prices.

Given the relationship between the prices of carbon emissions and energy, econometric models of the latent carbon emission prices are specified, estimated, evaluated, and used for forecasting carbon emissions prices for Japan, based on monthly data from December 2008 to April 2018. The econometric models show that, as sources of electricity, the logarithms of coal and oil, though not LNG, as well as a deterministic time trend and a tax change dummy variable, are statistically significant in explaining the logarithm of carbon emissions, with oil being more significant than coal.

\section{LITERATURE REVIEW}

In the sparse literature for Europe, most of which has been based on the European Union Emissions Trading Scheme, Daskalakis and Mrkellos (2009) developed an empirical and theoretical framework for intraphase and inter-phase futures pricing and hedging, and examined data from EEX, Nord Pool and Powernext to analyze whether electricity risk premia were affected by the emission allowance prices. Daskalakis et al. (2009) 
provided evidence from modelling $\mathrm{CO} 2$ emissions allowance prices and derivatives in Europe using data from the European Trading Scheme.

More recently, Bushnell et al. (2013) examined profiting from regulation, based on data from the European carbon market. Oestreich and Tsiakas (2015) analyzed carbon emissions and stock returns using data from the European Union Emissions Trading Scheme. Martin et al. (2016) examined the impacts of the European Union Emissions Trading Scheme on regulated firms from its inception for ten years.

A review of the admittedly sparse literature on carbon emissions prices in Europe and China, as well as a comprehensive analysis regarding the availability of price data at the domestic regional level, is analyzed in Chang et al. $(2018,2019)$. The authors discuss a number of developments in China since 2005. Chen (2005) evaluated the costs of mitigating carbon emissions in China. Gregg et al. (2008) analysed the emissions patterns of China as the world leader in carbon emissions from fossil fuel consumption and cement production. Li and Colombier (2009) viewed the managing of carbon emissions in China through building energy efficiency. Chang (2010) examined a multivariate causality test of carbon dioxide emissions, energy consumption and economic growth in China.

Nam et al. (2014) compared the synergy between pollution and carbon emissions control for China and the USA. Zhang et al. (2014) considered emissions trading in China in terms of progress and future prospects. Zhang et al. (2014) used evidence from the Shapley value model to understand regional allocations of carbon emissions quotas in China. Liu et al. (2015) reviewed China's carbon-emissions trading, and the challenges for the future. Tang et al. (2015) evaluated the carbon emissions trading scheme exploration in China using a multiagent-based model. Zhang (2015) reformulated the low-carbon green growth strategy in China. Xiong et al. (2017) compared the allowance mechanism of China's carbon trading pilots with alternative schemes in the EU and California.

There would seem to be even less research on pricing carbon emissions at an international level. Zhang and Sun (2016) applied a Full BEKK multivariate conditional volatility model to analyze the time-varying correlations and dynamic spillovers between the Euro carbon market and fossil fuel market (for caveats regarding the Full BEKK model, particularly regarding the lack of mathematical regularity conditions, invertibility, the absence of a likelihood function, and hence no valid asymptotic statistical properties, see Chang and McAleer (2019)). Chang et al. (2017) and Chang and McAleer (2019) use daily data for EU carbon emissions futures prices, US carbon emissions spot prices, and spot and futures prices of oil and coal, to analyze volatility spillovers and Granger causality.

\section{KLEMS PRODUCTION FUNCTION FOR CARBON EMISSIONS AND ENERGY}

Inputs are required to produce outputs, and the relationship is captured in a production function. A relatively broad specification is the KLEMS production function (OECD, 2001), which uses Capital (K), Labour (L), Energy (E), Materials (M), and Services (S) as the inputs into the production process. Typically, the most widely observable input is energy, which is based on the use of electricity. As a special case of KLEMS, consider the KLEM production function (see, for example, Lecca at al., 2011) that is given as: 


$$
C E=A K^{a 1} L^{a 2} E^{a 3} M^{a 4} \exp (u)
$$

where CE denotes the production of the output of Carbon Emissions, Capital $(K)$, Labour $(L)$, Energy or Electricity $(E)$, and Materials $(M)$ are the inputs, $A$ is a constant, the partial production parameters are assumed to satisfy constant returns to scale, and the random error term is assumed to be $u \sim i i d(0,1)$. CE and $E$ may be assumed to be proportional, with the random factor of proportionality given by:

$$
k=A K^{a 1} L^{a 2} M^{a 4} \exp (u)
$$

It is worth noting that $k$ is not restricted in its range, such as $(0,1)$. As $k$ has a unit of measurement, $k$ can take any value depending on the units of measure of the variables, such as:

$$
C E=k E^{a 3}
$$

If it is assumed that output prices are set according to an average cost pricing, the prices of $C E$ and $E$ may be approximated by a model with a constant factor of proportionality. This relationship be given as:

$$
P_{C E}=k P_{E}
$$

where $P_{C E}$ is the price of $C E, P_{E}$ is the price of energy (such as electricity), such that $k$ in equation (4) is the constant factor of proportionality. In practice, $k$ may be known in advance or may be determined empirically using appropriate modelling techniques.

\section{MODELLING LATENT CARBON EMISSION PRICES}

In the definitional relationship for a random variable, $\boldsymbol{Y}$, in equation (5), namely:

$$
Y=Y^{*}+\text { measurement error }
$$

where $Y$ is the measured value of the random variable, which is subject to measurement error, and $Y^{*}$ is the true value of the random variable, with no measurement error, the following four relationships are possible:

$$
\begin{array}{ll}
\text { (i) } & Y=P_{C E}, \quad Y^{*}=P_{C E}^{*} \\
\text { (ii) } & Y=P_{C E}, \quad Y^{*}=P_{E}^{*} \\
\text { (iii) } & Y=P_{E}, \quad Y^{*}=P_{E}^{*} \\
\text { (iv) } & Y=P_{E}, \quad Y^{*}=P_{C E}^{*}
\end{array}
$$$$
\text { (iii) } \quad Y=P_{E}, \quad Y^{*}=P_{E}^{*}
$$

where $P_{C E}$ and $P_{C E}^{*}$ denote the (possibly) observed prices of carbon emissions and its underlying true price, respectively, and $P_{E}$ and $P_{E}^{*}$ denote the observed and underlying true prices of energy, respectively.

For the sake of the argument, in case (iv) if the price of energy is stationary, $P_{E}$ can be regressed on a set of observable variables using OLS, as given in equation (6):

$$
P_{E}=b_{0}+b_{1} Z_{1}+b_{2} Z_{2}+b_{3} Z_{3}+\text { error }
$$

to yield estimated fitted values, as given in equation (7):

$$
\widehat{P_{E}}=\widehat{b_{0}}+\widehat{b_{1}} Z_{1}+\widehat{b_{2}} Z_{2}+\widehat{b_{3}} Z_{3}=\frac{1}{k} \widehat{P_{C E}^{*}}
$$

where $\widehat{b}_{\imath}$ denotes the OLS estimates of the unknown parameters $b_{i}(i=0,1,2,3)$, and $\widehat{P_{E}}$ denotes the estimated fitted values of energy prices from equation (6), which are equivalent to the estimated fitted values of carbon emissions, $\left(\widehat{P_{C E}^{*}}\right)$, adjusted by the factor $(1 / k)$ (see equation $\left.(4)\right)$, where $k$ may be known in advance 
or may be determined empirically. If energy prices are nonstationary rather than stationary, the price model for energy in equation (6) could be estimated using a cointegrating relationship or a vector autoregressive distributed lag model. Alternatively, prices could be transformed into log differences in prices, which are equivalent to the rate of growth in prices, and then estimated by OLS (in empirical finance, log differences in prices are referred to as rates of return).

\section{ESTIMATING LATENT CARBON EMISSION PRICES}

In what follows, the models presented in the previous section will be estimated using the available price and volume data to obtain estimated fitted latent prices of carbon emissions. It is assumed that there is a known expected relationship between carbon emissions and the generation of electricity using the data in cases (2) (12) above. This would be based on applied engineering practice and structural form modelling, as presented in the previous section, as follows:

$$
p_{0} q_{0}=k p_{1} q_{1} \exp (u)
$$

where $k$ is assumed to be a known factor of proportionality, and $u$ is assumed to be a random measurement error, possibly independently and identically distributed, with mean 0 and constant variance.

Equation (8) relates the unknown price and known quantity of carbon emissions to the known price and known quantity of energy (or electricity), which confirms that the resulting price equation refers directly to carbon emissions. Equation (9) is the logarithmic equivalent of equation (8). Let $p q=e$ (expenditure), with $k=1$, without loss of generality, so that equation (8) becomes:

$$
\log \left(e_{0}\right)=\log \left(e_{1}\right)+u
$$

It follows that:

$$
\mathrm{E}\left[\log \left(e_{0}\right)\right]=E\left[\log \left(e_{1}\right)\right]
$$

as $\mathrm{E}(u)=0$, such that the estimated fitted value of equation (10) is given as:

$$
\widehat{\log \left(e_{0}\right)}=\widehat{\log \left(e_{1}\right)}
$$

A regression of $\log \left(e_{1}\right)$ on $\log \left(e_{2}\right), \ldots, \log \left(e_{12}\right)$ gives:

$$
\log \left(e_{1}\right)=c+\delta_{2} \log \left(e_{2}\right)+\cdots+\delta_{12} \log \left(e_{12}\right)+\text { error }
$$

for which the estimated fitted value of $\log \left(e_{1}\right)$ is given as:

$$
\widehat{\log \left(e_{1}\right)}=\hat{c}+\widehat{\delta_{2}} \log \left(e_{2}\right)+\cdots+\widehat{\delta_{12}} \log \left(e_{12}\right)
$$

where $\hat{c}, \widehat{\delta_{2}}, \ldots, \widehat{\delta_{12}}$ are the OLS estimates of $c, \delta_{2}, \ldots, \delta_{12}$, respectively.

Recalling equation (11), it follows that:

$$
\left.\left.\widehat{\log \left(e_{0}\right.}\right)=\widehat{\log \left(e_{1}\right.}\right)=\hat{c}+\widehat{\delta_{2}} \log \left(e_{2}\right)+\cdots+\widehat{\delta_{12}} \log \left(e_{12}\right)
$$

which gives an "optimal" estimate of $\log \left(e_{0}\right)$.

As $\log \left(e_{0}\right)=\log \left(p_{0} q_{0}\right)$ and $\widehat{\log e_{0}}=\log \left(p_{0}\right)+\log q_{0}$, the prices of carbon emissions can be calculated "optimally" as:

$$
\widehat{\log \left(p_{0}\right)}=\widehat{\log \left(e_{0}\right)}-\log \left(q_{0}\right)
$$

This novel method would seem to be the first to calculate carbon emissions prices in theory, as well as in practice using monthly data for Japan. Further to the above discussion, if data were available for $p_{0}$, the 
price of energy (or electricity), as well as prices on some renewable and nonrenewable energy inputs, we could compare whether $Z_{1}$ jointly was more or less significant $Z_{z}$ aim explaining $p_{0}$.

\section{EMPIRICAL ESTIMATES AND ANALYSIS}

Two cases are considered, as follows:

Case 1: Seasonally unadjusted data, with a deterministic trend and dummy variable;

Case 2: Seasonally adjusted data, with a deterministic trend and dummy variable.

The dummy variable, which is introduced to accommodate the influence of increases in electricity prices on May 2017 arising from tax changes in Japan, equals 0 before September 2017, and 1 after September 2017. For both seasonally unadjusted and seasonally adjusted data, that is, Cases 1 and 2, the logarithms of coal and oil, as well as the deterministic time trend and the tax change dummy variable, are statistically significant in explaining the logarithm of carbon emissions, with oil being more significant than coal. The logarithm of LNG is not statistically significant at any conventional levels of significance in explaining the logarithm of carbon emissions in either Case 1 or Case 2. The forecasts of $\log (E)$ for seasonally unadjusted and seasonally adjusted data are shown in Figures 1(a) and 1(b), respectively, where two standard errors in each figure provide the $95 \%$ confidence intervals. In each case, there were noticeable falls in the forecasts of the logarithm of electricity from mid-2015 through to early-2016, and substantial rises toward the end of 2017.

The predicted values are based on the formulae for the logarithmic values in equation (16), with the predicted carbon emissions prices obtained using the exponential function for the seasonally unadjusted data, as follows:

$$
\begin{aligned}
& \log \left(\overline{C E}_{p}\right)=\widehat{\log (E)}-\log \left(E_{v o l}\right) \\
& \left.\widehat{C E}_{p}=\exp [\widehat{\log (E})-\log \left(\mathrm{E}_{\text {vol }}\right)\right]
\end{aligned}
$$

and for the seasonally adjusted data, as follows:

$$
\begin{aligned}
& \log \left(\widehat{C E_{p, S A}}\right)=\log \left(\widehat{E_{S A}}\right)-\log \left(E_{v o l, S A}\right) \\
& \widehat{C E_{p, S A}}=\exp \left[\log \left(E_{S A}\right)-\log \left(E_{v o l, S A}\right)\right]
\end{aligned}
$$

The patterns of the predicted carbon emissions prices in Figures 2(a) and 2(b) emulate the patterns described in Figures 1(a) and 1(b), with significant upward movements in both the seasonally unadjusted and seasonally adjusted data after mid-2017. The correlation between $\widehat{\log (E)}$ and $\overline{\log (E)} \widehat{S A}_{A}$ of 0.997 is given in Table 6, while the correlation between the seasonally unadjusted and seasonally adjusted predicted carbon emissions prices of 0.992 are given in Table 7. It is clear that the forecasts of carbon emission prices are very similar, regardless of whether seasonally unadjusted or seasonally adjusted data are used.

\section{CONCLUDING REMARKS}

It is widely accepted in the international scientific and global communities that climate change and global warming are significantly affected by carbon emissions that arise from the burning of fossil fuels, specifically coal, oil and gas. Although prices of fossil fuels are readily available, the prices of carbon emissions arising from competitive markets for the product are not yet commercially available. 
As carbon emissions can be taxed appropriately when accurate commercial market prices are known and accepted at an international level, carbon emissions can be taxed appropriately for purposes of measuring, capturing, storing and trading in carbon emissions at the regional, national, and international levels.

The paper used a novel KLEM production function approach to calculate the latent carbon emission prices, where carbon emissions are regarded as the output and capital (K), labour (L), energy (E) (or electricity), and materials (M), are the inputs into the production process. As the inputs $\mathrm{K}, \mathrm{L}$ and $\mathrm{M}$ are essentially fixed on a daily or monthly basis, or otherwise low frequency data observations, whereas $\mathrm{E}$ can be changed more frequently, such as daily or monthly, it follows that changes in carbon emissions are functions of changes in $\mathrm{E}$.

On the conditions that prices depend on average cost pricing, the prices of carbon emissions and energy may be approximated by an energy production model with a constant factor of proportionality, so that carbon emission prices will be a function of energy prices. Using this novel modelling approach, the paper estimated carbon emission prices for Japan using seasonally adjusted and unadjusted monthly data on the volumes of carbon emissions and energy, as well as energy prices, from December 2008 to April 2018.

The econometric models showed that, as sources of electricity, the logarithms of coal and oil, though not LNG, are statistically significant in explaining the logarithm of carbon emissions, with oil being more significant than coal. The models had high power in predicting the latent prices of carbon emissions.

The theoretical approach and empirical application developed in the paper should be useful for purposes of public policy debate and decision making. Accurate predictions of the latent prices of carbon emissions, and the imposition of appropriate environmental taxes, are essential in order to mitigate the effect of carbon emissions on global warming and climate change. 